Check for updates

Cite this: RSC Adv., 2019, 9, 35456

\title{
Comparison of two sequential extraction procedures for tungsten fractionation in the tungsten mining soils
}

\author{
Ruiping Li, (D)*ab Wenjie Wang, ${ }^{a}$ Shiliang Wang, ${ }^{a}$ Chunye Lin, ${ }^{\text {tb }}$ Xiangyi Wang, ${ }^{a}$ \\ Lingchuan Meng $^{a}$ and Xianlong Yuan ${ }^{c}$
}

Two sequential extraction procedures including Tessier and Wenzel schemes have been evaluated for the study of tungsten fractionation in soil samples adjacent to the World's largest and longest-operating tungsten mines in China. The efficiency and suitability of two methods and the corresponding extraction steps for partitioning tungsten were compared. Results showed the Tessier scheme classical for cation metals was inappropriate for tungsten fractionation. Although the percentage of readily bioavailable tungsten fractions extracted by the Tessier method is comparable to the Wenzel method, the Tessier scheme still has some drawbacks for partitioning tungsten mainly arising from the lack of selectivity of some of the reagents used. The Wenzel scheme has higher extraction recovery and efficiency than the Tessier method, especially for extracting amorphous and crystalline oxyhydroxides which were mainly responsible for tungsten retention. As a final conclusion, the study indicated that the Wenzel scheme should be more suitable for tungsten fractionation, but we need to make further improvement on the Wenzel scheme by supplementing the extraction stage for the oxidisable fraction to find a reliable and easy to use method to characterize tungsten forms in all soil samples to provide valuable information for risk assessment.

Received 6th September 2019 Accepted 28th October 2019

DOI: 10.1039/c9ra07158j

rsc.li/rsc-advances
At present, it has been widely recognized that the mobility, bioavailability, toxicity, migration and geo-chemical cycle of the heavy metal should not only depend on their total concentration but also on their specific chemical forms and on their binding state (precipitated with primary or secondary minerals, complexed by organic ligands, and so on). ${ }^{9,10}$ Sequential fractionation procedures (SEP) are commonly used to determine the solid phase forms of metals in soils. In this process, the soil or sediment sample is submitted to the selective reagents aiming to successively solubilize the different fractions of metals. Numerous sequential extraction procedures have been developed which mainly vary in the use of extracting reagents, target forms, and the order of separation of each particular form of metal. ${ }^{10}$ In contrast with the cationic nature of heavy metals, tungsten is predominantly present in soils and sediments as oxyanions. ${ }^{7,8}$ Hence, the feasibility of applying these sequential extraction schemes designed for cationic metals for fractionating soil tungsten should been questioned.

Few studies have reported the tungsten fraction in agricultural soils. The purpose of this research was to compare the extraction efficiencies of two operationally defined sequential extraction schemes, i.e. Tessier SEP classically for cationic metals and Wenzel SEP for oxyanions. This paper compares and discusses in detail the results of the fractionation of tungsten aiming to find the most appropriate SEP for tungsten and

\footnotetext{
${ }^{a}$ School of Geography and Tourism, Qufu Normal University, Rizhao Campus, Rizhao, China. E-mail: liruiping858@163.com; Tel: +86103980705

${ }^{b}$ State Key Joint Laboratory of Environmental Simulation and Pollution Control, School of Environment, Beijing Normal University, Beijing, China. E-mail: c.lin@bnu.edu.cn, Fax: +8610 58801858; Tel: +861058801858

${ }^{c}$ Rizhao Environmental Monitoring Station, Rizhao, China
} 
provide valuable information in the framework of risk assessment.

\section{Materials and methods}

\subsection{Soil source and its physicochemical properties}

The study site is situated in Dayu County, the southern Jiangxi province of southern China. It is characterized by subtropical monsoon climate, with average annual precipitation and temperature of $1591.5 \mathrm{~mm}$ and $18.5^{\circ} \mathrm{C}$, respectively. There are three major tungsten mines in Dayu County: Xihuashan, Dangping, and Piaotang, among which Xihuashan is the first tungsten mine operated in China. Tungsten occurs in wolframite $\left(\mathrm{Fe}, \mathrm{MnWO}_{4}\right)$ in these mines. Local farmers usually irrigate cultivated field with river water originated from the $\mathrm{W}$ mining area. Our previous study found that average total tungsten concentration in agricultural soil there reached up to $47.39 \mathrm{mg} \mathrm{kg}{ }^{-1}$, revealing a great potential risks for human health. ${ }^{\mathbf{1 1 , 1 2}}$

The sampling points are set according to the distribution of tungsten mining and tungsten processing enterprises. 140 topsoil samples (about 0 to $20 \mathrm{~cm}$ depth) were collected in the agricultural fields adjacent to $\mathrm{W}$ mines and factory. The samples were collected from four to five locations at a given location and then mixed to make composite samples to improve site representation. 31 soils differing in the level of tungsten contamination were selected to extract tungsten fractionation with two sequential extraction procedures (SEPs). For all samples, the $\mathrm{pH}$, organic matter (OM), and the contents of $\mathrm{Al}$, $\mathrm{Fe}, \mathrm{Mn}$ and $\mathrm{W}$ were measured. The specific experimental methods and instruments have been reported in our previous studies. $^{12}$

\subsection{Extraction of tungsten in soil samples}

The Tessier and the Wenzel sequential extraction schemes were used in this study. The detailed tungsten extraction procedures defined by each SEP are briefly listed in Table 1 . The selective sequential extractions were conducted in centrifuge tubes (polypropylene, $50 \mathrm{~mL}$ ). The quantities indicated below refer to $1 \mathrm{~g}$ of the soil samples (dry weight of the original sample used for the initial extraction). After each successive extraction, separation was performed by centrifuging at $10000 \mathrm{rpm}$ for $10 \mathrm{~min}$ using a Xiang Yi centrifuge at room temperature. The supernatants were then removed with a pipette and filtered through a $0.45 \mu \mathrm{m}$ filter membrane. For each SEP, the residual soils from the previous step were washed using de-ionized water and again centrifuged for $10 \mathrm{~min}$. The washing water was added to the extract for measurement. The $\mathrm{W}$ concentration in each solution was determined using ICP-MS (X Series II, Thermo Electron) and with ICPAES (IRIS Intrepid II, Thermo Electron). The corresponding limit of detection (LOD) is $0.02 \mu \mathrm{g} \mathrm{L}^{-1}$ and $0.015 \mathrm{mg} \mathrm{L}^{-1}$, respectively. The linearity of the calibration curve was acceptable when the linear regression coefficient $\left(r^{2}\right)$ was $\geq 0.998$.

Reagent blanks, duplicate and standard reference materials (GSD7a, Chinese Academy of Measurement Sciences) were used to assess the quality assurance and control. The control sample was analyzed for every 10 samples. The average relative errors (triplicate) of standard reference materials were 3.6 (1.9-5.8)\%, $4.0(3.5-4.5) \%$, $3.9(3.2-4.7) \%$, and $3.2(6.2-2.0) \%$ for $\mathrm{Al}, \mathrm{Fe}, \mathrm{Mn}$, and $\mathrm{W}$, respectively.

\section{Results and discussion}

\subsection{Total tungsten concentration and physicochemical characterization of the soil samples}

Fig. 1 summarized some mineral matrix elements, the levels of $\mathrm{pH}$ and total tungsten in the soil samples collected in the agricultural fields near the tungsten mines. The $\mathrm{pH}$ values of the soil were in the range 4.92-5.92 with averages of 5.39, showing its acidic property. The amount of organic matter content (OM) was $2.33-7.06 \%$. The $\mathrm{Mn}, \mathrm{Al}$, and Fe levels in the soil ranged from $345.12-2123.89 \mathrm{mg} \mathrm{kg}^{-1}, 4.66-17.52 \%$, and

Table 1 Steps in the selective sequential extraction procedure for tungsten in the soil

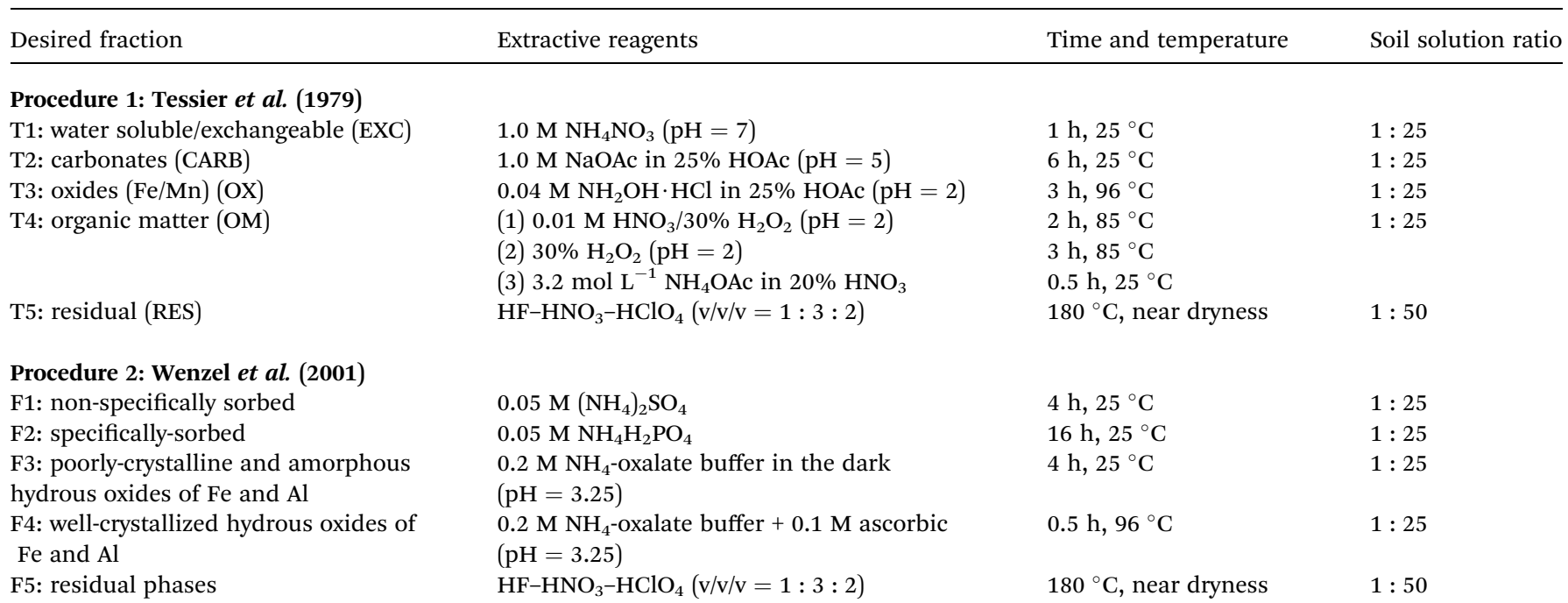





Fig. 1 Distribution histogram of contents of mineral matrix elements and total tungsten in the soil, the outlier box plot is shown in the figure.

1.33-7.25\%, respectively. The mean contents of $\mathrm{Al}, \mathrm{Fe}, \mathrm{Sc}$ and Mn were generally higher than their background contents in the soils of the Jiangxi province and China. ${ }^{\mathbf{1 3 , 1 4}}$ The soil in the area is generally classified as ferrosols in Chinese taxonomy (oxisols). ${ }^{\mathbf{1 5}}$

The average total concentration of $\mathrm{W}$ in all the surface agricultural soil samples ranged from $2.81 \mathrm{mg} \mathrm{kg}^{-1}$ to $794.58 \mathrm{mg} \mathrm{kg}^{-1}$, with a mean concentration of $61.70 \mathrm{mg} \mathrm{kg}^{-1}$. Total tungsten concentration in the soil at all the sampling sites was exceeded the soil background level of China and world, ${ }^{\mathbf{1 3}, \mathbf{1 4}}$ and mean content of tungsten was 11.68 times high as the background value of Jiangxi province. This result implied that the surface agricultural soil adjacent to tungsten mines in Dayu
County was polluted by tungsten and revealed great potential risks to environment and human health.

\subsection{Chemical partitioning of tungsten in the soil samples by two SEPs}

3.2.1. Method of Tessier. The chemical partitioning results of $\mathrm{W}$ obtained by two SEPs are presented in Fig. 2. Tungsten recovery of Tessier procedure ranges from $83.7 \%$ to $114.4 \%$ for all the samples studied, which can be regarded as acceptable. Although this procedure was designed for evaluating the behavior of cationic metal species, it has also been successfully used for fractionation of oxyanions such as arsenic., ${ }^{\mathbf{9 1 0} 16}$ 


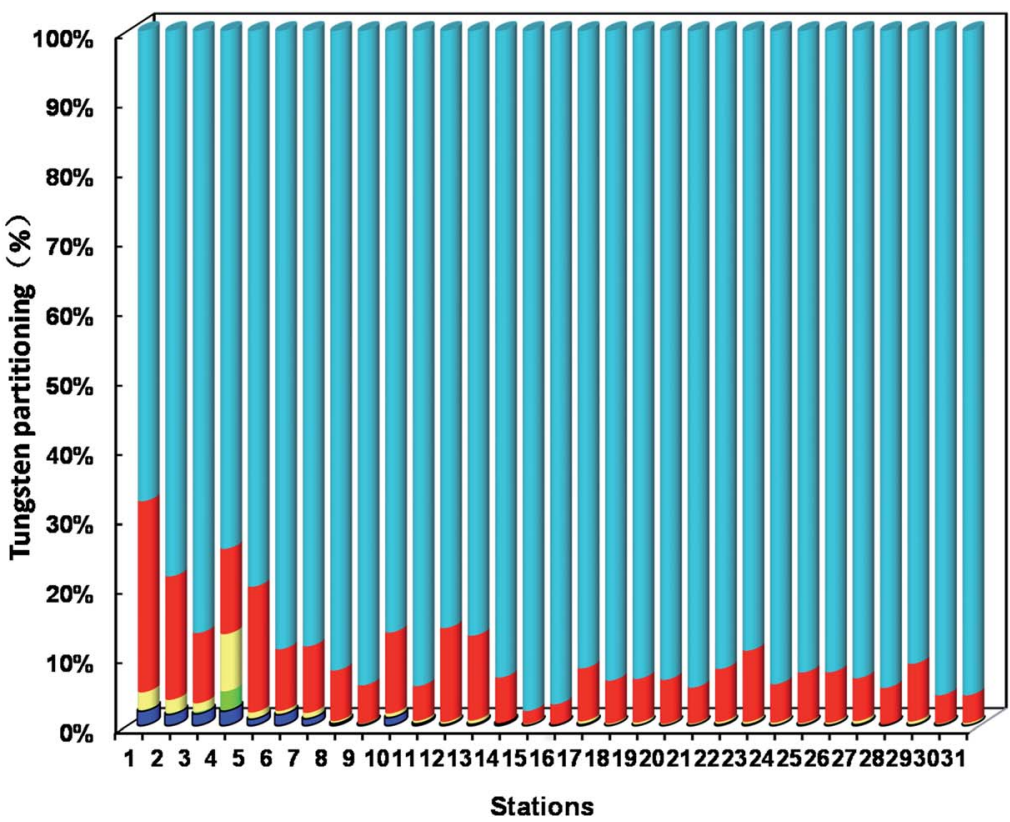

\title{
A) procedure of Tessir
}

\author{
- T5: residual \\ घ4: oxidizable \\ T3: reducible \\ T2: carbonate bond \\ $\square T 1$ : exchangeable and soluble
}

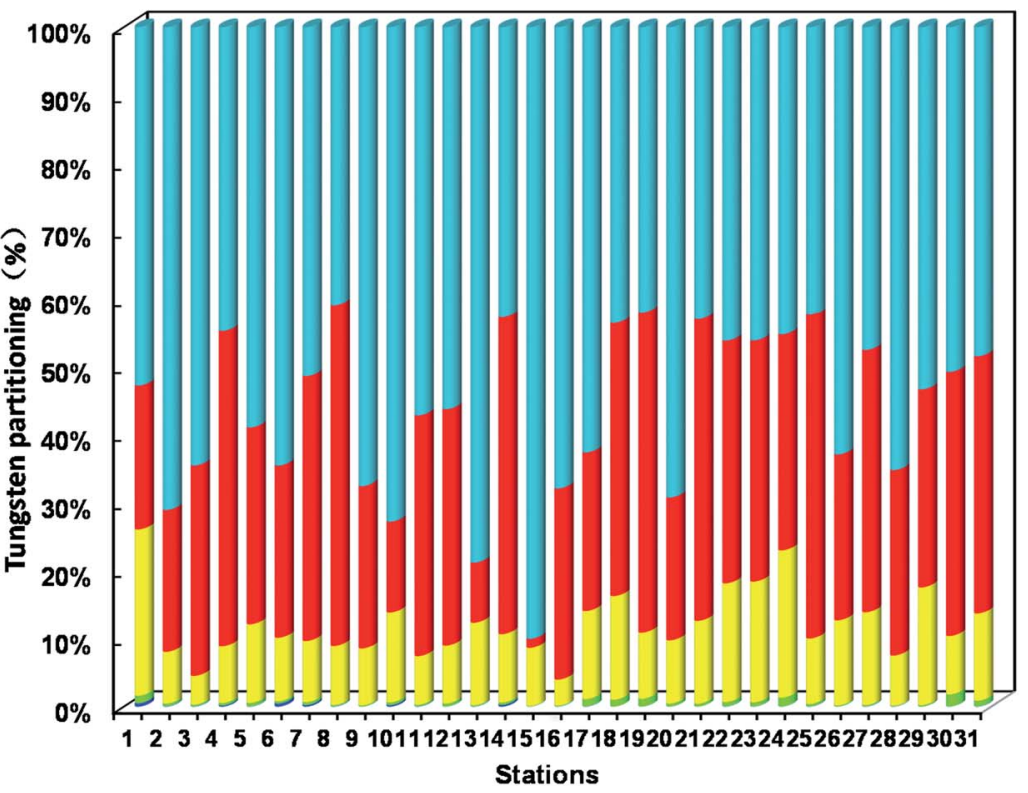

\section{B) procedure of Wenzel}

\author{
F5: residual \\ E4: crystalline Fe and Al oxides bound \\ F3: amorphous Fe and Al oxides bound \\ F2: specifically sorbed \\ E1: non-specifically sorbed
}

Fig. 2 Tungsten partitioning (\%) in the soil studied by use of two sequential extraction procedures: (A) procedure of Tessier; (B) procedure of Wenzel (mean \pm standard deviation, $n=3$ ).

Therefore, the feasibility of applying this sequential extraction schemes for the study of soil fractionation of $\mathrm{W}$ has been questioned and evaluation is necessary.

The first fraction $\mathrm{T} 1$ usually contains water-soluble tungsten fraction made up of free ions and ions complexed with soluble constituents, and also includes weakly adsorbed tungsten species retained on the soil surface by relatively weak electrostatic interactions and tungsten that can be released by ionexchange processes. Therefore, this fraction represents the most mobile and potentially the most bioavailable tungsten species in the environment. The amount of tungsten leached in T1 ranges from $0.02 \%-2.07 \%$ in soil samples.
Fraction T2 considers the tungsten precipitated or coprecipitated with carbonate. This fraction is sensitive to $\mathrm{pH}$ changes. The percentages of tungsten extracted by this fraction have large variations among soils studied, ranging from $0.01 \%$ to $2.82 \%$.

In the third fraction $\mathrm{T} 3$ step, the reducible fraction, which theoretically is represented the content of tungsten bound to iron and manganese oxides. Because hydrous oxides of manganese and iron are thermodynamically unstable under the anoxic circumstances, the relative tungsten concentration extracted by T3 varies greatly, from $0.09 \%$ to $8.26 \%$.

The fourth fraction T4 is supposed to be the tungsten associated with $\mathrm{OM}$ and sulfides. For this fraction, tungsten may be 
incorporated in many forms of organic matter such as living organisms, detritus or coatings on mineral particles. Percentage of tungsten leached in T4 ranges from $1.84 \%-27.49 \%$, second only to that leached in the residual fraction.

The residual tungsten is determined in the fifth fraction T5. A relative much high percentage of tungsten was noticed in the residual fraction for all the studied soil samples due to the historically high tungsten contamination, ranging from $67.75 \%$ to $97.94 \%$.

Generally, the percentages of $\mathrm{W}$ associated with different fraction are in the order of residual $(90.02 \%)>$ organic matter $(8.64 \%)>$ Fe-Mn oxides $(0.76 \%)>$ exchangeable and soluble $(0.45 \%)>$ carbonate $(0.13 \%)$. This result is consistent with the results of Wilson and Pyatt, ${ }^{17}$ who investigated tungsten species in the acidic spoils adjacent to an abandoned tungsten mine by BCR scheme, showing about $99 \%$ of tungsten in the residual fraction.

3.2.2. Method of Wenzel. Tungsten recovery by this method ranges from $97.3 \%$ to $107.2 \%$, which generally is also satisfactory.

This scheme results in a more differentiated pattern of $\mathrm{W}$ distribution (Fig. 2) than the method of Tessier because it is intended to distinguish among important species of tungsten in soil samples.

Tungsten in the first fraction F1 is readily labile tungsten and may represent the most important fraction related to the environment risks. Similar to the previous SEP, this tungsten fraction accounts approximately $0.49 \%$ in samples.

As regards the specifically adsorbed tungsten fraction F2, percentage of tungsten leached is relatively small, ranging from $0.10 \%-1.77 \%$. The amount of this species is closely correlated with the change of the soil solution $\mathrm{pH}$.

F3 fraction contains the tungsten co-precipitated mainly with amorphous iron and aluminum (hydr)oxides. A relatively significant part of the total tungsten is associated with F3, ranging from $3.79 \%$ to $24.45 \%$.

The fourth fraction step (F4) is designed to leach the fraction bound to crystalline iron and aluminum (hydr)oxides. A higher mobilization rate of tungsten is extracted by F4, ranging from $20.88 \%$ to $47.70 \%$ for almost all soil samples except site 15 (1.31\%), site $13(8.83 \%)$ and site $10(13.37 \%)$, respectively.

In contrast with the previous scheme, tungsten extracted in the residual form F5 is generally low, ranging from $40.88 \%-$ $78.87 \%$ except site 15 (90.08\%). However, this fraction is also the main chemical specie of $\mathrm{W}$ in soil samples.

Finally, this scheme is proposed to be more specific to evaluate the fractionation of anionic metal species, such as As and Se. Partitioning of tungsten in the soil are in the order of residual $(56.50 \%)>$ well-crystalline hydrous oxides of iron and aluminum $(31.55 \%)>$ poorly-crystalline hydrous oxides of iron and aluminum $(11.37 \%)>$ non-specifically adsorbed fraction $(0.50 \%)$ and specifically adsorbed fraction $(0.08 \%)$. This result is different from the Tessier method, especially the tungsten fraction bound to iron and aluminum (hydr)oxides.

\subsection{Comparison between the two extraction schemes}

To facilitate the comparison between the two extraction methods, the extraction steps were classified into four groups: (i) exchangeable and acid soluble fraction, (ii) reducible fraction (Fe-, Al- and Mn-oxyhydroxides), (iii) oxidizable fraction (organic matter and sulphides), and (iv) residual fraction. The chemical partitioning of tungsten (\%) of different fractions by two SEPs in the soils is summarized in Fig. 2. The comparison results of two SEPs for equivalent groups are shown in Fig. 3. Two SEPs were compared for equivalent fractions across all soil samples using the $t$-test for paired samples.

The exchangeable and acid soluble fractions show the amount of tungsten that is a loosely bound phase and liable to change with environmental conditions. On the whole, the extraction results for the exchangeable/carbonates fraction indicate that two extraction methods are performing similarly, but the exchangeable/carbonates fraction extracted by Wenzel SEP (F1 + F2) for most samples is significantly higher $(p<0.01)$ than that extracted by the corresponding fraction of Tessier SEP $(\mathrm{T} 1+\mathrm{T} 2)$ (Fig. 3). One reason may be that the Wenzel SEP has a sufficiently long extraction interval (reaction times of 4 and 16 h) (Table 1). On the other hand, $\left(\mathrm{NH}_{4}\right)_{2} \mathrm{SO}_{4}$ had been shown to extract anionic species slightly more effective than the acetate solutions of equal ionic strength, ${ }^{18}$ and had also been successfully used to extract exchangeable As and Se from soils. ${ }^{18,19}$ At the same time, phosphate solutions may be efficient in extracting tungsten from soils because of competitive effect for adsorption sites on natural soil and other minerals (such as ferrihydrite, kaolinite and oxisols). ${ }^{\mathbf{1 2 , 2 0 , 2 1}}$ Generally, heavy metals in the exchangeable and acid soluble fractions are considered readily and potentially bioavailable species that is useful in risk assessment of metal leaching to the groundwater. Thus, in terms of the extraction efficiency of bioavailable species, the method of Wenzel would be better than the Tessier SEP becoming an essential tool for the risk assessment of tungsten polluted sites.

However, the tungsten contents bound to iron and manganese oxides extracted by two schemes are quite different (Fig. 3). The Wenzel SEP $(\mathrm{F} 3+\mathrm{F} 4)$ produces significantly higher $(p<$ 0.01 ) concentrations bound to iron and manganese (about 2-11 times) for all soil samples than that extracted by the



Fig. 3 Tungsten partitioning profiles in soil according to the procedure of Tessier and the procedure of Wenzel (mean \pm standard deviation, $n=3$ ). 
corresponding fraction of Tessier SEP (T3) (Fig. 3). It has been reported that the oxides/hydroxides of $\mathrm{Al}$, Fe and $\mathrm{Mn}$ in environmental solid samples are the main tungsten scavengers. ${ }^{21-23}$ In principle, the reducible fraction could be split into three fractions: easily reducible fraction (Mn oxides); moderately reducible fraction (amorphous Fe oxides); and poorly-reducible fraction (crystalline Fe oxides). The relatively high concentration of $\mathrm{Al}$ and $\mathrm{Fe}$ in the study soil samples may lead to a high proportion of reducible components of tungsten. Therefore, the relatively low percentages of reducible tungsten extracted by Tessier SEP (T3) may be due to the insufficient extraction efficiency by hydroxylamine hydrochloride in acetic acid medium. Hydroxylamine hydrochloride is rather a specific reagent of $\mathrm{Mn}$ oxides, and less than $5 \%$ of Fe oxides were reported to be dissolved under the conditions used. ${ }^{24-26}$ So hydroxylamine hydrochloride is used to leach the easily reducible fraction (Mn oxides) and the moderately reducible fraction (amorphous Fe oxides), ${ }^{27}$ which is not a sufficiently reductive step. However, it is reported that ammonium oxalate in acid medium is effective for extracting amorphous oxides/hydroxides of both $\mathrm{Fe}$ and Al. ${ }^{\mathbf{9} 10,28}$ In the absence of catalyzing effect of light, this reagent does not dissolve crystalline Fe oxides. ${ }^{\mathbf{9} 28}$ At the same time, the ascorbic acid/ammonium oxalate reagent adequate for dissolving crystalline Fe oxides and does not attack silicates. ${ }^{\mathbf{9 1 0}, 28}$ Therefore, Wenzel SEP is likely to advantageous over the Tessier SEP to extract the reducible fraction of tungsten concentration associated with $\mathrm{Fe} / \mathrm{Al}$ oxides/hydroxides.

The oxidizable fraction theoretically represents the contents of metals bound to organic matter and sulfides. However, the soil in the study area is generally classified as ferrosols in Chinese taxonomy (oxisols). The secondary minerals in the oxisols mainly included kaolinite, vermiculite, hydromica, and hematite. ${ }^{15}$ Organic matter and sulfides are relatively scarce in this area. That is why we choose the Wenzel methods with no reducible fraction. Tessier SEP uses hydrogen peroxide and ammonium acetate in acidic medium to extract the oxidation of tungsten fraction bounding to organic material and sulfides, and extract low but obvious concentrations of tungsten (Fig. 3). This result could be due to the inefficiency of previous extraction steps. In particular, the fact is probably related to the incompletely dissolved of hematite in previous extraction steps. ${ }^{15}$ Filgueiras et $a l .{ }^{9}$ reported that hydrogen peroxide can attack the amorphous Fe/Mn oxides and has being used occasionally prior to leaching the moderately reducible fraction.

Although the tungsten content in the silicate fractions are environmentally insignificant, knowledge of the tungsten release by this extraction step is relatively important, because it indicates the efficiency of the previous extraction steps. Even though the extraction reagent in this step of the two SEPs is identical, the extraction results are different because of the efficiency of previous extraction steps. In particular, the percentage of tungsten in the residual fraction extracted by Tessier SEP (90.02\% of the average tungsten extracted) is higher $(p<0.01)$ than that by Wenzel SEP $(56.50 \%$ of the average tungsten extracted) (Fig. 3). This fact is probably due to the late dissolution of the mineral phases such as hematite tungstate, which was not completely leaching during the previous extraction steps with an inadequate efficiency of extraction agents used.

\section{Conclusion}

This work was intended to stress the importance of selection of the most appropriate sequential extraction scheme for study of the distribution of tungsten in soil samples, because the sequential extraction procedures provide noteworthy information about mobility of tungsten, bioavailability and eco-toxicity so as to assess contamination risk. Then, two sequential extraction schemes were compared for tungsten fractionation in soil samples adjacent to the World's largest and longestoperating tungsten mine in China. Comparison between the different schemes showed that the results were highly dependent on the procedure used.

The classical Tessier scheme which are commonly used for cation metals, are not as appropriate for evaluation of tungsten fractionation, because some of the reagents used in this SEP, such as $\mathrm{NH}_{2} \mathrm{OH} \cdot \mathrm{HCl}$ and $\mathrm{H}_{2} \mathrm{O}_{2}$, are lack of selectivity and suitability leads to incompletely leaching the target tungsten fractions, such as $\mathrm{Fe} / \mathrm{Al}$ associated tungsten, and overestimate residual tungsten giving a far too low risk assessment.

The present study has showed that a high reliability of results is obtained by applying the Wenzel procedure. Because of the finer division of the main species in soil retaining tungsten, the reagents used are more selective for the desired target phases. The concentration of tungsten was extracted from the crystalline Fe oxide which may become bioavailable forms, was relegated to the residual phase. Therefore, the application of this scheme may enable differentiation of the most mobilizable forms of tungsten and provide interesting environmental results.

\section{Conflicts of interest}

There are no conflicts to declare.

\section{Acknowledgements}

The authors gratefully acknowledge the financial support for this work by the Ministry of Education Humanities and Social Sciences Research Youth Fund Project (No. 19YJCZH085), the National Natural Science Foundation of China (No. 41371441 and No. 41701562) and College Students Innovation, and Entrepreneurship Training Program (No. 201810446039).

\section{References}

1 EPA, Emerging-Contaminant-Tungsten, U.S. EPA 505-F-07-005, 2008.

2 A. D. R. Kelly, M. Lemaire, Y. K. Young, J. H. Eustache, C. Guilbert, M. F. Molina and K. K. Mann, Toxicol. Sci., 2013, 131, 434-446.

3 R. Lemus and C. F. Venezia, Crit. Rev. Toxicol., 2015, 45, 388411. 
4 A. J. Bednar, R. E. Boyd, W. T. Jones, C. J. McGrath, D. R. Johnson, M. A. Chappell and D. B. Ringelberg, Chemosphere, 2009, 75, 1049-1056.

5 D. Dermatas, W. Braida, C. Christodoulatos, N. Strigul, N. Panikov, M. Los and S. Larson, Environmental Forensics, 2004, 5, 5-13.

6 G. S. Tuna and W. Braida, Soil Sediment Contam., 2014, 23, 838-849.

7 S. C. Hsu, H. L. Hsieh, C. P. Chen, C. M. Tseng, S. C. Huang, C. H. Huang, Y. T. Huang, V. Radashevsky and S. H. Lin, J. Hazard. Mater., 2011, 189, 193-202.

8 A. Koutsospyros, W. Braida, C. Christodoulatos, D. Dermatas and N. Strigul, J. Hazard. Mater., 2006, 136, 1-19.

9 A. V. Filgueiras, I. Lavilla and C. Bendicho, J. Environ. Monit., 2002, 4, 823-857.

10 C. Gleyzes, S. Tellier and M. Astruc, Trends Anal. Chem., 2002, 6, 451-467.

11 R. P. Li, R. N. Luan, C. Y. Lin, D. Q. Jiao and B. B. Guo, $R S C$ Adv., 2014, 4, 63875-63881.

12 C. Y. Lin, R. P. Li, H. G. Cheng, J. Wang and X. Shao, PLoS One, 2014, 9, e91981.

$13 \mathrm{H}$. Bowen, Environmental chemistry of the elements, Academic, New York, 1979, p. 333.

14 F. S. Wei, J. S. Chen, Y. Y. Wu, C. J. Zheng and D. Z. Jiang, Background contents of elements in China soils, Publishing House of Chinese Environmental Sciences, Beijing, 1990.

15 J. L. He, G. Y. Xu, H. M. Zhu and G. H. Peng, Background contents of elements in the soils of Jiangxi Province,
Publishing House of Chinese Environmental Sciences, Beijing, 2006.

16 M. Mihaljevic, M. Ponavic, V. Ettler and O. Sebek, Anal. Bioanal. Chem., 2003, 377, 723-729.

17 B. Wilson and F. B. Pyatt, Sci. Total Environ., 2006, 370, 401408.

18 S. E. Johnston and W. M. Barnard, Soil Sci. Soc. Am. J., 1979, 43, 304-308.

19 K. Saeki and S. Matsumoto, Commun. Soil Sci. Plant Anal., 1994, 25, 2147-2158.

20 R. P. Li, C. Y. Lin and X. T. Liu, RSC Adv., 2016, 6, 1987219877.

21 N. Xu, C. Christodoulatos, A. Koutsospyros and W. Braida, Land Contam. Reclam., 2009, 17, 45-57.

22 H. Hur and R. J. Reeder, J. Colloid Interface Sci., 2016, 461, 249-260.

23 S. Rakshit, B. Sallman, A. Davantes and G. Lefevre, Chemosphere, 2017, 168, 685-691.

24 T. T. Chao, Soil Sci. Soc. Am. J., 1972, 36(5), 764-768.

25 C. Gleyzes, S. Tellier, R. Sabrier and M. Astruc, Environ. Technol., 2001, 22, 27-38.

26 A. Suda, T. Makino and T. Higashi, Geoderma, 2011, 163, 291-295.

27 G. Kalyvas, D. Gasparatos and I. Massas, Arch. Agron. Soil Sci., 2018, 64, 1549-1563.

28 A. Lock, D. Wallschlager, C. McMurdo, L. Tyler, N. Belzile and G. Spiers, Environ. Pollut., 2016, 219, 1102-1108. 